\title{
Tratamento de sementes industrial: potencial de armazenamento de sementes de soja tratadas
}

Elisa Lemes, Andreia Almeida, Adilson Jauer, Filipe Mattos, Lilian Tunes

Universidade Federal de Pelotas - UFPel. E-mail: lemes.elisa@yahoo.com.br

\begin{abstract}
Resumo
Visto que a cada ano são lançados novos produtos para o tratamento de sementes, pesquisas que visem determinar o desempenho de sementes de soja tratadas com diferentes combinações de produtos ao longo do armazenamento apresentam relevância para o mercado de sementes. Diante disso, objetivou-se avaliar o efeito do tratamento de sementes com diferentes agroquímicos durante o armazenamento de duas cultivares de soja. Foram conduzidos estudos independentes para cada cultivar, utilizando o delineamento experimental inteiramente casualizado com esquema de parcelas subdivididas, sendo a parcela constituída pela combinação dos produtos no tratamento das sementes (T1- Maxim Advanced; T2Maxim Advanced + Cruiser; T3- Cruiser + Amulet + Maxim Advanced; T4- Avicta + Cruiser + Maxim Advanced; T5- Maxim Advanced + Cruiser + A17960; T6- Cropstar + Derosal Plus; T7- Standak Top; T8Dermacor + Derosal Plus; T9- Dermacor + Derosal Plus + Cruiser; T10- Cruiser Opti + Maxim Advanced) e a subparcela composta pelos períodos de armazenamento (0, 45, 90, 135 e 180 dias). O potencial fisiológico das sementes tratadas foi avaliado por meio do teste de germinação, teste de envelhecimento acelerado e emergência em canteiro. Com base nos resultados obtidos, conclui-se que as combinações de produtos com thiametoxam em sua formulação apresenta, no geral, menor redução da qualidade fisiológica ao longo do armazenamento. As sementes tratadas com os produtos combinados de Imidacloprido+Tiodicarbe e Carbendazim+Thiram reduzem o potencial fisiológico das sementes ao longo do período de armazenamento.
\end{abstract}

Palavras-chave: Glycine max (L.) Merrill; recobrimento de sementes; vigor; protetores de sementes.

\section{Industrial seed treatment: storage potential of soybean seeds treated}

\begin{abstract}
Since new seed treatment products are launched each year, research aimed at determining the soybean seeds treated performance with different combinations of these products throughout the storage are important for the seed market. The objective of this study was to evaluate the effect of seed treatment with different products during the storage of two soybean cultivars. Independent studies were conducted for each cultivar, using a completely randomized experimental design in a split plots. The plot consisted of the combination of the products in the seed treatment ((T1- Maxim Advanced; T2- Maxim Advanced + Cruiser; T3- Cruiser + Amulet + Maxim Advanced; T4- Avicta + Cruiser + Maxim Advanced; T5- Maxim Advanced + Cruiser + A17960; T6- Cropstar + Derosal Plus; T7- Standak Top; T8- Dermacor + Derosal Plus; T9- Dermacor + Derosal Plus + Cruiser; and T10- Cruiser Opti + Maxim Advanced) and the subplot constituting of storage periods $(0,45,90,135$ and 180 days). The physiological quality evaluation of treated seeds was carried out through germination, accelerated aging, and emergence tests. Based on the results obtained, it is concluded that the combinations of products with thiamethoxam in its formulation show, in general, lower reduction of the physiological quality throughout the storage. Seeds treated with the combined products of Imidacloprid + Thiodicarb and Carbendazim + Thiram reduce the physiological potential of the seeds over the storage period.
\end{abstract}

Keywords: Glycine max (L.) Merrill; seed coating; vigor; germination. 


\section{Introdução}

A soja é uma cultura amplamente utilizada para a elaboração de rações animais, produção de óleo e outros subprodutos, além do seu consumo in natura que tem se expandido nos dias atuais. Nesse contexto, o Brasil é o segundo maior produtor de soja, sendo a cultura de maior destaque na agricultura brasileira, que ocupa mais de $50 \%$ da área total, correspondendo em torno de 35,7 milhões de hectares semeadas (CONAB, 2019).

O sucesso do potencial produtivo deste e de outros cultivos faz-se necessário aprimorar as técnicas de produção, afim de buscar alternativas para que se atinja o máximo de produção com o mínimo de impacto ambiental possível. Desta forma, o uso de sementes de alta qualidade, aliada ao emprego de produtos que auxiliem o desempenho destas no campo, é quesito fundamental para se obter o estande inicial adequado de plantas. De acordo com Kolchinski et al. $(2005 ; 2006)$, sementes de soja com alta qualidade fisiológica irão proporcionar plantas com maiores taxas de crescimento inicial e eficiência metabólica, além de maior área foliar, maior produção de matéria seca e maiores rendimentos, aumentando assim as chances de sucesso da lavoura.

Vários fatores podem limitar 0 rendimento da cultura da soja, como doenças e pragas, que podem ocorrer durante todo o ciclo da planta e estarem associadas às sementes. Neste sentido, algumas tecnologias utilizadas, como o tratamento de sementes, ajudam a controlar os avanços das doenças e as infestações de insetos (FREITAS, 2011). Esta é uma prática consolidada e economicamente recomendada para soja, desde que utilizados produtos ou misturas de produtos de forma adequada, na dosagem correta e distribuídos uniformemente em todo o lote de sementes (AVELAR et al., 2011). Com a incorporação do tratamento industrial de sementes (TSI) às linhas de beneficiamento de grande parte das empresas produtoras de sementes, pode-se ter maior aproveitamento dos insumos, sendo um processo realizado independente das condições climáticas, uma vez que o TSI é realizado em unidades agroindustriais apropriadas (GADOTTI; OLIVEIRA, 2014). Outro fator de elevada importância na manutenção da qualidade fisiológica das sementes, tratadas ou não com inseticidas, é o armazenamento, o qual visa garantir a qualidade das sementes durante o período compreendido entre o beneficiamento e comercialização (PESKE et al., 2012).

Neste sentido, há a necessidade de aprofundar o conhecimento sobre o potencial fisiológico de sementes de soja após o tratamento químico e o armazenamento (DAN et al., 2012), e tendo em vista a semente como principal insumo e alicerce da produção, os atributos da qualidade (fisiológicos e sanitários, principalmente) necessitam andar juntos. Desta forma, o tratamento químico além de assegurar a sanidade do lote, garante todo potencial genético que este carrega, de modo que a escolha do produto e os testes de controle devem evitar riscos de danos ao potencial fisiológico das sementes (NUNES, 2016).

Algumas pesquisas têm mostrado que o uso de certos produtos no tratamento de sementes pode ocasionar redução na germinação e na sobrevivência de plântulas quando avaliados em testes de germinação ao longo do armazenamento de sementes de milho e soja (FESSEL et al., 2003; DAN et al., 2010), entretanto, outras afirmam que o mesmo não interfere ou propicia maior porcentagem de germinação (PIRES et al., 2004; PEREIRA et al., 2005; AVELAR et al., 2011; LUDWIG et al., 2015). Diante dessa controvérsia de resultados e visto que a cada ano são lançados novos produtos para o tratamento de sementes, objetivou-se avaliar o efeito de diferentes agroquímicos para o tratamento de sementes ao longo do armazenamento de sementes de duas cultivares de soja.

\section{Material e Métodos}

O trabalho foi realizado no Laboratório Didático de Análise de Sementes (LDAS), pertencente ao Programa de Pós-Graduação em Ciência e Tecnologia de Semente da Universidade Federal de Pelotas (FAEM/UFPel). Foram utilizadas sementes de soja das cultivares SYN 1059 RR (V-Top) e SYN 13561 IPro.

As sementes de soja foram tratadas usando tratadora Niklas com rotação de $25 \mathrm{~Hz}$ e ciclo de aplicação de 15 minutos $(5 \mathrm{~min}$ de injeção, 8 min misturando, 2 min descarga), constituindo 10 tratamentos (Tabela 1). Em seguida ao tratamento, as sementes foram submetidas à secagem ambiente por um período de 24 horas. Posteriormente, estas foram imediatamente avaliadas pelos testes de germinação, envelhecimento acelerado e emergência em canteiro, sendo considerado a 
época zero, e em seguida, as mesmas foram acondicionadas em sacos de papel e armazenadas em câmara com condições controladas (umidade relativa de $40 \%$ e temperatura de $16^{\circ} \mathrm{C}$ ); as sementes foram avaliadas após 45, 90, 135 e 180 dias de armazenamento.

Tabela 1. Identificação dos produtos, concentrações e doses utilizadas no tratamento de sementes de soja pertencentes às cultivares.

\begin{tabular}{|c|c|c|c|c|}
\hline Tratamento & Produto Comercial & Ingrediente ativo & Concentração (g i.a) & $\begin{array}{c}\text { Dose utilizada } \\
\text { ( } \mathrm{mL} 100 \mathrm{~kg}^{-1} \text { de sementes) }\end{array}$ \\
\hline T1 & Maxim Advanced & Fludioxonil & 25 & 100 \\
\hline \multirow{2}{*}{$\mathrm{T} 2$} & Maxim Advanced & Fludioxonil & 25 & 100 \\
\hline & Cruiser & Thiametoxam & 350 & 250 \\
\hline \multirow{3}{*}{ T3 } & Cruiser & Thiametoxam & 350 & 200 \\
\hline & Amulet & Fipronil & 250 & 100 \\
\hline & Maxim Advanced & Fludioxonil & 25 & 100 \\
\hline \multirow{3}{*}{ T4 } & Avicta & Abamectina & 500 & 100 \\
\hline & Cruiser & Thiametoxam & 350 & 200 \\
\hline & Maxim Advanced & Fludioxonil & 25 & 100 \\
\hline \multirow{3}{*}{ T5 } & Maxim Advanced & Fludioxonil & 25 & 100 \\
\hline & Cruiser & Thiametoxam & 350 & 250 \\
\hline & A17960 & Cyantraniliprole & 600 & 80 \\
\hline \multirow{4}{*}{ T6 } & Cropstar & Imidacloprido & 150 & \multirow{2}{*}{500} \\
\hline & & Tiodicarbe & 450 & \\
\hline & Derosal Plus & Carbendazim & 150 & \multirow{2}{*}{200} \\
\hline & & Thiram & 350 & \\
\hline \multirow{3}{*}{$\mathrm{T7}$} & Standak Top & Fipronil & 250 & \multirow{3}{*}{200} \\
\hline & & Piraclostrobina & 25 & \\
\hline & & Tiofanato Metilico & 225 & \\
\hline \multirow{3}{*}{$\mathrm{T} 8$} & Dermacor & Clorantraniliprole & 625 & 100 \\
\hline & Derosal Plus & Carbendazim & 150 & \multirow{2}{*}{200} \\
\hline & & Thiram & 350 & \\
\hline \multirow{4}{*}{ T9 } & Dermacor & Clorantraniliprole & 625 & 100 \\
\hline & Derosal Plus & Carbendazim & 150 & \multirow{2}{*}{200} \\
\hline & & Thiram & 350 & \\
\hline & Cruiser & Thiametoxam & 350 & 200 \\
\hline \multirow{3}{*}{ T10 } & Cruiser Opti & Thiametoxam & 210 & \multirow{2}{*}{500} \\
\hline & & LambdaCyhalotrin & 37,5 & \\
\hline & Maxim Advanced & Fludioxonil & 25 & 100 \\
\hline
\end{tabular}

O potencial fisiológico das sementes foi avaliado por meio das seguintes avaliações em cada período de armazenamento:

Teste de germinação (G): conduzido com 200 sementes, subdivididas em quatro subamostras de 50 sementes. O substrato utilizado foi rolo de papel do tipo "germitest", previamente umedecido com água destilada, na proporção de 2,5 vezes a massa do papel seco. Após a semeadura, os rolos de papel foram mantidos em germinador, à temperatura de $25^{\circ} \mathrm{C}$. As avaliações foram efetuadas aos oito dias após a semeadura, conforme as Regras para Análise de Sementes (BRASIL, 2009). Os resultados foram expressos em porcentagem de plântulas normais.

Teste de envelhecimento acelerado (EA): executado em caixas acrílicas do tipo "gerbox" compostas de tela metálica. Foram adicionados 40 $\mathrm{mL}$ de água destilada ao fundo de cada caixa, sendo as sementes distribuídas de forma uniforme 
e em camada única, sobre a tela suspensa. Em seguida, as caixas, contendo as sementes, foram tampadas e acondicionadas em câmara BOD, à temperatura de $41^{\circ} \mathrm{C}$, pelo período de 48 horas. Decorrido o tempo, as sementes foram submetidas ao teste de germinação, sendo a avaliação realizada aos cinco dias após a semeadura (MARCOS FILHO, 2015). Os resultados foram expressos em porcentagem de plântulas normais.

Emergência em canteiro (EC): foram semeadas 200 sementes em canteiro distribuídas em quatro linhas de 50 sementes. Após a semeadura, a umidade do solo foi mantida na capacidade de campo. A avaliação foi realizada em contagem única, aos 21 dias após a semeadura, sendo os resultados expressos em porcentagem de plântulas emergidas (NAKAGAWA, 1999).

Foram conduzidos estudos independentes para cada cultivar, utilizando o delineamento experimental inteiramente casualizado com esquema de parcelas subdivididas, sendo a parcela constituída pela combinação dos produtos no tratamento das sementes e a subparcela composta pelos períodos de armazenamento $(0,45,90,135$ e 180 dias). Os dados foram analisados quanto à normalidade e homocedasticidade, se não atendidas as condições, foram realizadas as transformações de dados necessárias e, posteriormente, submetidos à análise de variância $(p<0,05)$. Sendo significativas pelo teste " $F$ ", as médias da combinação dos produtos do tratamento de sementes foram comparadas pelo teste de Scott-Knott e o período de armazenamento submetido à análise de regressão polinomial, todos a $5 \%$ de probabilidade. Para análise estatística utilizou-se o software $R^{\odot}(R$ CORE TEAM, versão 3.3.1, 2016).

\section{Resultados e Discussão}

Observou-se que as diferentes combinações de produtos (TS) e períodos de armazenamento (PA), assim como a interação entre eles apresentaram efeito significativo sobre a germinação e o vigor determinado pelo teste de envelhecimento acelerado e emergência em canteiro, para as ambas as cultivares de soja (Tabela 2).

Tabela 2. Resumo da ANOVA empregando-se o delineamento em parcelas subdivididas para a porcentagem de plântulas normais obtidas nos testes de germinação (G), envelhecimento acelerado (EA) e emergência em canteiro (EC) das cultivares de soja SYN 1059 RR (V-Top) e SYN 13561 IPro.

\begin{tabular}{lccccccc}
\hline & \multicolumn{4}{c}{ SYN 1059 RR } & \multicolumn{3}{c}{ SYN 13561 IPro } \\
\cline { 2 - 8 } \multicolumn{1}{c}{ Fonte de Variação } & GL & G & EA & EC & G & EA & EC \\
\hline TS & 9 & $*$ & $*$ & $*$ & $*$ & $*$ & $*$ \\
Resíduo A (RA) & 30 & & & & & & \\
\hline Parcela & 39 & & & & & & \\
\hline PA (PA) & 4 & $*$ & $*$ & $*$ & $*$ & $*$ & $*$ \\
TS*PA & 36 & $*$ & $*$ & $*$ & $*$ & $*$ & $*$ \\
Resíduo B (RB) & 120 & & & & & & \\
\hline C.V RA (\%) & & 1,03 & 1,33 & 1,04 & 1,45 & 1,02 & 1,30 \\
C.V RB (\%) & & 1,07 & 1,37 & 0,93 & 1,09 & 1,02 & 0,91 \\
\hline
\end{tabular}

* ou ns = significativo e não significativo, respectivamente, a $5 \%$ de probabilidade pelo teste $\mathrm{F}$. TS= fator tratamento de sementes; $\mathrm{PA}=$ fator períodos de armazenamento; $\mathrm{GL}=\mathrm{Grau}$ de liberdade; $\mathrm{CV}=$ Coeficiente de variação.

O tratamento 6, que consistiu na combinação dos produtos Cropstar + Derosal Plus, foi o que proporcionou menor porcentagem de plântulas normais obtidas no teste de germinação, envelhecimento acelerado e emergência em campo em todos os períodos de armazenamento, sendo este fato constatado para as cultivares testadas (Tabela 3 ). 0 tratamento de sementes pode provocar alterações degenerativas no metabolismo, bem como desencadear processo de desestruturação das membranas celulares das sementes, provocando redução da qualidade fisiológica (PICCININ et al., 2013). Ainda, a qualidade das sementes tratadas e armazenadas pode ser influenciada pelo produto químico empregado no tratamento das mesmas e, também, da cultivar utilizada (TONIN et al., 2014).

No geral, a mistura de produtos contendo tiametoxam na formulação foram os que 
resultaram em melhor desempenho das sementes da cultivar SYN 1059 RR (V-Top) e SYN 13561 IPro. Almeida et al. (2014), verificaram que o tratamento de sementes de soja com tiametoxam contribui para que a perda de vigor das sementes determinada pelo armazenamento seja mais lenta quando comparada com sementes sem tratamento. No entanto, Piccinin et al. (2013) observaram que o tratamento de sementes de soja cultivar BMX Magna RR, BMX Potência RR, NK 3358 RR e NK 7059 RR, com os inseticidas fipronil e thiamethoxam prejudicou a qualidade fisiológica destas cultivares quando submetidas ao armazenamento por 180 dias em condições normais de armazenamento.

Tabela 3. Germinação (G), envelhecimento acelerado (EA) e emergência a campo (EC) de sementes de soja das cultivares SYN 1059 RR (V-Top) e SYN 13561 IPro tratadas com vários produtos.

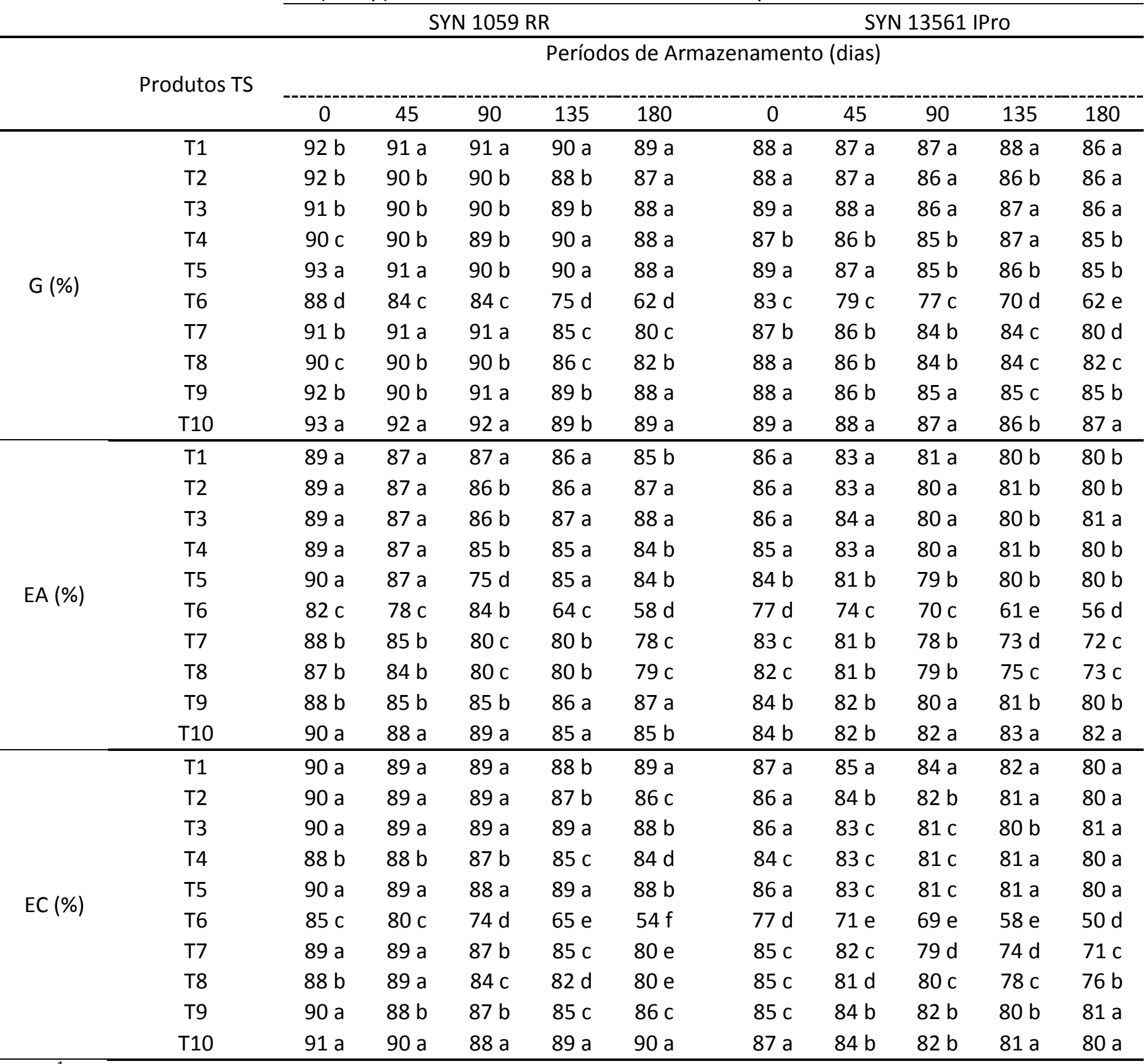

${ }^{1}$ Médias seguidas por mesma letra minúscula na coluna, em cada período de armazenamento, não diferem entre si pelo teste de Scott-Knott $(p<0,05)$. T1- Maxim Advanced; T2- Maxim Advanced + Cruiser; T3- Cruiser + Amulet + Maxim Advanced; T4- Avicta + Cruiser + Maxim Advanced; T5- Maxim Advanced + Cruiser + A17960; T6- Cropstar + Derosal Plus; T7- Standak Top; T8- Dermacor + Derosal Plus; T9- Dermacor + Derosal Plus + Cruiser; T10- Cruiser Opti + Maxim Advanced. 
Para a germinação, envelhecimento acelerado e emergência em campo verificou-se que o armazenamento influenciou o desempenho das sementes tratadas, independente do produto utilizado (Tabela 4). Houve redução da germinação das sementes conforme 0 prolongamento do período de armazenamento, em relação ao vigor das sementes tratadas avaliadas através do envelhecimento acelerado e emergência em campo, os dados se ajustaram aos modelos linear e quadrático negativo, sendo observado as maiores reduções na porcentagem de plântulas normais pelo tratamento 6 . Em contraste, Conceição (2013), não verificou prejuízos com o tratamento de sementes de soja composto pelas combinações de fungicida carbendazin + thiram e inseticida imidacloprid + tiodicarbe sobre a qualidade fisiológica das sementes de soja cultivar NA 4823 RG, BMX Turbo RR e Fundacep 62 RR ao longo do período de armazenamento de 8 meses de armazenamento em condição ambiente.
Tem sido evidenciado que alguns produtos, quando aplicados isoladamente ou em combinação, podem, em determinadas situações, ocasionar redução na qualidade fisiológica das sementes, devido ao efeito de fitotoxicidade (PEREIRA et al., 2011). Marcos Filho e Souza (1983), avaliando o comportamento de sementes de soja tratadas com fungicidas e armazenadas durante seis meses, concluíram que o tratamento das sementes antes do armazenamento favorece a manutenção do vigor das sementes. No entanto, Cardoso et al. (2004) e Krohn e Malavasi (2004) relataram que sementes tratadas com fungicidas e armazenadas apresentaram melhor desempenho nos períodos iniciais de armazenamento e, após quatro meses, apresentaram redução da qualidade fisiológica, provavelmente devido ao efeito fitotóxico do tratamento químico. 
Tabela 4. Regressões dos períodos de armazenamento para germinação (\%), envelhecimento acelerado (\%) e emergência a campo (\%) de sementes de soja, cultivar SYN 1059 RR (V-Top) tratadas com vários produtos.

\begin{tabular}{|c|c|c|c|c|c|c|c|}
\hline Variável & Produtos TS & $\begin{array}{c}\text { Equação } \\
Y= \pm a x^{2} \pm b x \pm c\end{array}$ & $P\left(b_{i x}\right)$ & $\mathrm{R}^{2}$ & PMET & YE & DDZ \\
\hline \multirow{10}{*}{ G } & $\mathrm{T} 1$ & $-0,01556 x+92$ & $*$ & 0,96 & 180 & 89 & -3 \\
\hline & $\mathrm{T} 2$ & $-0,02667 x+92$ & $*$ & 0,95 & 180 & 87 & -5 \\
\hline & T3 & $-0,01611 x+91$ & $*$ & 0,96 & 180 & 88 & -3 \\
\hline & $\mathrm{T} 4$ & $-0,00778 x+90$ & $*$ & 0,48 & 180 & 88 & -2 \\
\hline & T5 & $-0,02278 x+93$ & $*$ & 0,88 & 180 & 89 & -4 \\
\hline & T6 & $-0,136 x+91$ & $*$ & 0,86 & 180 & 67 & -24 \\
\hline & T7 & $-0,062 x+93$ & $*$ & 0,79 & 180 & 82 & -11 \\
\hline & T8 & $-0,044 x+92$ & $*$ & 0,78 & 180 & 84 & -8 \\
\hline & T9 & $-0,02111 x+92$ & $*$ & 0,85 & 180 & 88 & -4 \\
\hline & T10 & $-0,02389 x+93$ & $*$ & 0,89 & 180 & 89 & -4 \\
\hline \multirow{10}{*}{ EA } & $\mathrm{T} 1$ & $-0,02 x+89$ & $*$ & 0,92 & 180 & 85 & -4 \\
\hline & $\mathrm{T} 2$ & $0,00027 x^{2}-0,06 x+89$ & $*$ & 0,99 & 111 & 86 & -3 \\
\hline & T3 & $0,00028 x^{2}-0,06 x+89$ & $*$ & 0,96 & 107 & 86 & -3 \\
\hline & T4 & $-0,02667 x+88$ & $*$ & 0,90 & 180 & 83 & -5 \\
\hline & T5 & $0,00092 x^{2}-0,19 x+91$ & $*$ & 0,54 & 103 & 81 & -10 \\
\hline & T6 & $-0,138 x+86$ & $*$ & 0,72 & 180 & 61 & -25 \\
\hline & $\mathrm{T7}$ & $-0,056 x+87$ & $*$ & 0,90 & 180 & 77 & -10 \\
\hline & T8 & $-0,044 x+86$ & $*$ & 0,87 & 180 & 78 & -8 \\
\hline & T9 & $0,00032 x^{2}-0,059 x+88$ & $*$ & 0,87 & 92 & 85 & -3 \\
\hline & T10 & $-0,03 x+90$ & $*$ & 0,87 & 180 & 85 & -5 \\
\hline \multirow{10}{*}{$\mathrm{EC}$} & T1 & $0,00011 x^{2}-0,03 x+90$ & * & 0,77 & 136 & 88 & -2 \\
\hline & $\mathrm{T} 2$ & $-0,02 x+90$ & $*$ & 0,96 & 180 & 86 & -4 \\
\hline & T3 & $-0,00889 x+90$ & $*$ & 0,80 & 180 & 88 & -2 \\
\hline & T4 & $-0,02444 x+89$ & $*$ & 0,92 & 180 & 85 & -4 \\
\hline & T5 & $-0,00889 x+90$ & $*$ & 0,58 & 180 & 88 & -2 \\
\hline & T6 & $-0,171 x+87$ & $*$ & 0,97 & 180 & 56 & -31 \\
\hline & $\mathrm{T7}$ & $-0,0489 x+90$ & $*$ & 0,86 & 180 & 81 & -9 \\
\hline & T8 & $-0,05222 x+89$ & $*$ & 0,89 & 180 & 79 & -10 \\
\hline & T9 & $-0,0244 x+89$ & $*$ & 0,82 & 180 & 87 & -2 \\
\hline & T10 & $0,00025 x^{2}-0,05 x+91$ & * & 0,85 & 100 & 88 & -3 \\
\hline
\end{tabular}

$\mathrm{P}=$ Probabilidade a $5 \%$ ( Significativo e ${ }^{\text {ns }}$ não significativo). PMET = Ponto de máxima eficiência teórica. YE= Valor estimado. DDZ= Diferença da dose zero; T1- Maxim Advanced; T2- Maxim Advanced + Cruiser; T3- Cruiser + Amulet + Maxim Advanced; T4- Avicta + Cruiser + Maxim Advanced; T5- Maxim Advanced + Cruiser + A17960; T6- Cropstar + Derosal Plus; T7- Standak Top; T8- Dermacor + Derosal Plus; T9- Dermacor + Derosal Plus + Cruiser; T10- Cruiser Opti + Maxim Advanced.

Foi observado tendência semelhante das regressões da germinação entre as cultivares SYN 1059 RR (V-Top) e SYN 13561 IPro (Tabela 4 e 5), com redução linear, variando entre 3 e 24 e 2 e 20 pontos percentuais, respectivamente, em relação a dose zero. Para a porcentagem de plântulas normais obtidas pelos testes de vigor (envelhecimento acelerado e emergência a campo, foram verificadas reduções mais acentuadas no potencial fisiológico dessas sementes, uma vez que o tratamento 6 apresentou redução de 25 e 31 pontos 
percentuais para a cultivar SYN 1059 RR (V-Top) e de 22 e 26 pontos percentuais para a cultivar SYN 13561 IPro. Isso demonstra que independentemente da combinação dos produtos utilizados, a viabilidade e o vigor das sementes ao longo do armazenamento é prejudicada.

Tabela 5. Regressões dos períodos de armazenamento para germinação (\%), envelhecimento acelerado (\%) e emergência a campo (\%) de sementes de soja, cultivar SYN 13561 IPro tratadas com vários produtos.

\begin{tabular}{|c|c|c|c|c|c|c|c|}
\hline & Produtos TS & $\begin{array}{c}\text { Equação } \\
Y= \pm a x^{2} \pm b x \pm c\end{array}$ & $P\left(b_{i x}\right)$ & $\mathrm{R}^{2}$ & PMET & YE & DDZ \\
\hline \multirow{10}{*}{ G } & $\mathrm{T1}$ & & NS & & & & \\
\hline & $\mathrm{T} 2$ & $-0,012 x+88$ & * & 0,63 & 180 & 86 & -2 \\
\hline & T3 & $-0,012 x+88$ & $*$ & 0,75 & 180 & 86 & -2 \\
\hline & T4 & & NS & & & & \\
\hline & T5 & $-0,017 x+88$ & * & 0,60 & 180 & 85 & -3 \\
\hline & T6 & $-0,112 x+84$ & $*$ & 0,95 & 180 & 64 & -20 \\
\hline & $\mathrm{T7}$ & $-0,0355 x+87$ & $*$ & 0,88 & 180 & 81 & -6 \\
\hline & T8 & $-0,0317 x+88$ & * & 0,93 & 180 & 82 & -6 \\
\hline & T9 & $-0,0161 x+87$ & $*$ & 0,66 & 180 & 84 & -3 \\
\hline & T10 & $-0,015 x+89$ & $*$ & 0,68 & 180 & 86 & -3 \\
\hline \multirow{10}{*}{ EA } & $\mathrm{T} 1$ & $-0,0328 x+85$ & $*$ & 0,83 & 180 & 79 & -6 \\
\hline & $\mathrm{T} 2$ & $-0,031 x+85$ & $*$ & 0,77 & 180 & 79 & -6 \\
\hline & T3 & $-0,0289 x+85$ & $*$ & 0,70 & 180 & 80 & -5 \\
\hline & T4 & $-0,025 x+84$ & $*$ & 0,79 & 180 & 80 & -4 \\
\hline & T5 & $0,00033 x^{2}-0,08 x+84$ & $*$ & 0,89 & 121 & 79 & -5 \\
\hline & T6 & $-0,122 x+78$ & $*$ & 0,97 & 180 & 56 & -22 \\
\hline & $\mathrm{T7}$ & $-0,07 x+84$ & $*$ & 0,96 & 180 & 71 & -13 \\
\hline & T8 & $-0,056 x+83$ & $*$ & 0,98 & 180 & 73 & -10 \\
\hline & T9 & $-0,02 x+83$ & * & 0,77 & 180 & 79 & -4 \\
\hline & $\mathrm{T} 10$ & $0,000132 x^{2}-0,029 x+83$ & $*$ & 0,42 & 110 & 81 & -2 \\
\hline \multirow{10}{*}{ EC } & $\mathrm{T} 1$ & $-0,03722 x+87$ & $*$ & 0,99 & 180 & 80 & -7 \\
\hline & $\mathrm{T} 2$ & $-0,03222 x+85$ & $*$ & 0,96 & 180 & 79 & -6 \\
\hline & T3 & $-0,0261 x+84$ & $*$ & 0,71 & 180 & 79 & -5 \\
\hline & T4 & $-0,0222 x+84$ & $*$ & 0,89 & 180 & 80 & -4 \\
\hline & T5 & $-0,0294 x+85$ & $*$ & 0,85 & 159 & 79 & -6 \\
\hline & T6 & $-0,147 x+78$ & $*$ & 0,95 & 180 & 52 & -26 \\
\hline & $\mathrm{T7}$ & $-0,077 x+85$ & * & 0,98 & 180 & 71 & -14 \\
\hline & T8 & $-0,04444 x+84$ & * & 0,97 & 180 & 76 & -8 \\
\hline & T9 & $-0,0256 x+85$ & $*$ & 0,81 & 180 & 80 & -5 \\
\hline & T10 & $-0,0367 x+86$ & $*$ & 0,92 & 180 & 79 & -7 \\
\hline
\end{tabular}

$\mathrm{P}=$ Probabilidade a $5 \%$ ( ${ }^{*}$ Significativo $\mathrm{e}^{\text {ns }}$ não significativo). $\mathrm{PMET}=$ Ponto de máxima eficiência teórica. $\mathrm{YE}=$ Valor estimado. DDZ= Diferença da dose zero. T1- Maxim Advanced; T2- Maxim Advanced + Cruiser; T3- Cruiser + Amulet + Maxim Advanced; T4- Avicta + Cruiser + Maxim Advanced; T5- Maxim Advanced + Cruiser + A17960; T6- Cropstar + Derosal Plus; T7- Standak Top; T8- Dermacor + Derosal Plus; T9- Dermacor + Derosal Plus + Cruiser; T10- Cruiser Opti + Maxim Advanced. 


\section{Conclusões}

As combinações de produtos com thiametoxam em sua formulação apresenta, no geral, menor redução da qualidade fisiológica ao longo do armazenamento.

As sementes tratadas com os produtos combinados de Imidacloprido+Tiodicarbe e Carbendazim+Thiram reduzem o potencial fisiológico das sementes ao longo do período de armazenamento.

\section{Referências}

ALMEIDA, A. S.; CASTELLANOS C. I. S.; DEUNER C.; BORGES C. T.; MENEGHELLO G. E. Efeitos de inseticidas, fungicidas e biorreguladores na qualidade fisiológica de sementes de soja durante o armazenamento. Revista de Agricultura, v.89, n.3, p.172-182, 2014. http://www.fealq.org.br/ojs/index.php/revistade agricultura/article/view/173

ARAÚJO, M. M. Caracterização e seleção de linhagens de soja resistentes ou tolerantes à ferrugem asiática. 2009. Dissertação (Mestrado) - Escola Superior de Agricultura Luiz de Queiroz, Piracicaba, 2009.

AVELAR, S. A. G.; BAUDET L.; PESKE S. T.; LUDWIG M. P.; RIGO G. A.; CRIZEL R. L.; OLIVEIRA S. Storage of soybean seed treated with fungicide, insecticide and micronutrient and coated with a liquid and powdered polymer. Ciência Rural, v.41, n.10, p.1719-1725, 2011. http://dx.doi.org/10.1590/S0103$\underline{84782011005000130}$

BRASIL. Ministério da Agricultura, Pecuária e Abastecimento. Regras para análise de sementes. Brasília: Mapa/ACS, 2009. 399 p.

CARDOSO, P. C.; BAUDET L.; PESKE S. T.; LUCCAFILHO, O. A. Armazenamento em sistema a frio de sementes de soja tratadas com fungicida. Revista Brasileira de Sementes, v.26, n.1, p.1523, 2004. http://dx.doi.org/10.1590/S0101$\underline{31222004000100003}$

CONAB. Acompanhamento da Safra Brasileira de Grãos 2018/2019 - Sétimo Levantamento Abril/2019, v.6 - Brasília: Conab, 2019.

CONCEIÇÃO, G. M. Tratamento químico de sementes de soja: qualidade fisiológica, sanitária e potencial de armazenamento. 2013, $53 \mathrm{f}$.
Dissertação (Mestrado em Agronomia) Universidade Federal de Santa Maria, Santa Maria, 2013.

DAN, L. G. M.; DAN H. A.; BARROSO A. L. L.; BRACCINI A. L. Qualidade fisiológica de sementes de soja tratadas com inseticidas sob efeito do armazenamento. Revista Brasileira de Sementes, v.32, n.2, p.131-139, 2010. http://dx.doi.org/10.1590/S0101$\underline{31222010000200016}$

DAN, L. G. M.; DAN H. A.; PICCININ G. G.; RICCI T. T.; ORTIZ A. H. T. Tratamento de sementes com inseticida e a qualidade fisiológica de sementes de soja. Revista Caatinga, v.25, n.1, p.45-51, 2012.

https://periodicos.ufersa.edu.br/index.php/caati nga/article/view/2073

FESSEL, S. A.; MENDONÇA E. A. F.; CARVALHO R. V.; VIEIRA R. D. Efeito do tratamento químico sobre a conservação de sementes de milho durante 0 armazenamento. Revista Brasileira de Sementes, v.25, n.1, p.25-28, 2003. http://www.scielo.br/pdf/rbs/v25n1/19626.pdf. https://doi.org/10.1590/S0101$\underline{31222003000100005}$

FREITAS, M. C. M. A cultura da soja no Brasil: o crescimento da produção brasileira e o surgimento de uma nova fronteira agrícola. Enciclopédia Biosfera, v.7, 12p, 2011. https://www.conhecer.org.br/enciclop/2011a/ag rarias/a\%20cultura\%20da\%20soja.pdf

GADOTTI, G.I.; OLIVEIRA, A.L. Aspectos técnicos e legais relacionados ao tratamento de sementes. Revista Seed News, v.19, n.3, p.34-36, 2014.

KOLCHINSKI, E. M.; SCHUCH L. O. B.; PESKE S. T. Crescimento inicial de soja em função do vigor das sementes. Revista Brasileira de Agrociência, v.12, n.2, p.163-166, 2006.

KOLCHINSKI, E. M.; SCHUCH L. O. B.; PESKE S. T. Vigor de sementes e competição intra-específica em soja. Ciência Rural, v.35, n.6, p.1248-1256, $2005 . \quad$ http://dx.doi.org/10.1590/S010384782005000600004

KROHN, N. G.; MALAVASI M. M. Qualidade fisiológica de sementes de soja tratadas com fungicidas durante e após o armazenamento. 
Revista Brasileira de Sementes, v.26, n.2, p.9197, 2004. http://dx.doi.org/10.1590/S0101$\underline{31222004000200013}$

LUDWIG, M. P.; OLIVEIRA S.; AVELAR S. A. G.; ROSA M. P.; LUCCA FILHO O. A.; CRIZEL R. L. Armazenamento de sementes de soja tratadas e seu efeito no desempenho de plântulas. Tecnologia e Ciência Agropecuária, v.9, n.1, p.5156 , 2015.

http://revistatca.pb.gov.br/edicoes/volume-092015/volume-9-numero-1-marco2015/tca9110.pdf

MARCOS FILHO, J. Fisiologia de sementes de plantas cultivadas. Londrina: ABRATES, 2015. 659 p.

MARCOS-FILHO, J.; SOUZA F. H. D. Conservação de sementes de soja tratadas com fungicidas. Anais Escola Superior de Agricultura "Luiz de Queiroz", v.40, n.1, p.181-201, 1983. https://doi.org/10.1590/S0071$\underline{12761983000100010}$

NAKAGAWA, J. Testes de vigor baseados no desempenho das plântulas. In: KRZYZANOWSKI, F. C.; VIEIRA, R. D.; FRANÇA-NETO, J. B. Vigor de sementes: conceitos e testes. Londrina: Abrates, 1999. Cap. 2, p. 9-13.

NUNES, J. C. S. Tratamento de sementes de soja como um processo industrial no Brasil. Revista SEED News, v.20, p.26-32, 2016.

PEREIRA, C. E.; GUIMARÃES R. M.; VIEIRA A. R.; EVANGELISTA J. R. E.; OLIVEIRA G. E. Tratamento fungicida e peliculização de sementes de soja submetidas ao armazenamento. Ciência e Agrotecnologia, v.35, n.1, p.158-164, 2011. http://dx.doi.org/10.1590/S1413-

$\underline{70542011000100020}$

PEREIRA, C.E. OLIVEIRA J. A.; GUIMARÃES R. M.; VIEIRA A. R.; SILVA J. B. C. Condicionamento fisiológico e revestimento de sementes de pimentão. Revista Ciência Agronômica, v.36, n.1, p.74-81, 2005. http://www.ccarevista.ufc.br/seer/index.php/cca revista/article/view/10

PICCININ, G. G.; BRACCINI, A. L.; DAN L. G. M.; BAZO G. L.; LIMA L. H. S. Influência do armazenamento na qualidade fisiológica de sementes de soja tratadas com inseticidas. Ambiência, v.9, n.2, p.289-298, 2013. https://revistas.unicentro.br/index.php/ambienci a/article/view/1462.

https://doi.org/10.5777/ambiencia.2013.02.04

PIRES, L.L.; BRAGANTINI C.; COSTA J.L.S. Armazenamento de sementes de feijão revestidas com polímeros e tratadas com fungicidas. Pesquisa Agropecuária Brasileira, v.39, n.7, p.709-715, 2004. http://dx.doi.org/10.1590/S0100204X2004000700013.

$R$ CORE TEAM. $\mathbf{R}$ - A language and environment for statistical computing. Vienna, Austria: $R$ Foundation for Statistical Computing. 2016. Disponível em: http://r-project.org. Acesso em: 17 ago. 2016.

TONIN, R. F. B.; LUCCA FILHO O. A.; BAUDET L. M. L.; ROSSETO M. Potencial fisiológico de sementes de milho híbrido tratadas com inseticidas e armazenadas em duas condições de ambiente. Scientia Agropecuaria, v.5, n.1, p.7-16, 2014. http://www.scielo.org.pe/scielo.php?script=sci a rttext\&pid=S2077-99172014000100001. https://doi.org/10.17268/sci.agropecu.2014.01.0 1 